Marquette University

e-Publications@Marquette

School of Dentistry Faculty Research and

Publications

Dentistry, School of

$12-2019$

\title{
A Glassy Carbon Electrode Modified with Reduced Graphene Oxide and Gold Nanoparticles for Electrochemical Aptasensing Of Lipopolysaccharides from Escherichia Coli Bacteria
}

\author{
Mehrab Pourmadadi \\ Shahid Beheshti University \\ Javad Shabani Shayeh \\ Shahid Beheshti University \\ Meisam Omidi \\ Shahid Beheshti University \\ Fatemeh Yazdian \\ University of Tehran \\ Masoud Alebouyeh \\ Shahid Beheshti University
}

See next page for additional authors

Follow this and additional works at: https://epublications.marquette.edu/dentistry_fac

Part of the Dentistry Commons

\section{Recommended Citation}

Pourmadadi, Mehrab; Shayeh, Javad Shabani; Omidi, Meisam; Yazdian, Fatemeh; Alebouyeh, Masoud; and Tayebi, Lobat, "A Glassy Carbon Electrode Modified with Reduced Graphene Oxide and Gold Nanoparticles for Electrochemical Aptasensing Of Lipopolysaccharides from Escherichia Coli Bacteria" (2019). School of Dentistry Faculty Research and Publications. 362.

https://epublications.marquette.edu/dentistry_fac/362 


\section{Authors}

Mehrab Pourmadadi, Javad Shabani Shayeh, Meisam Omidi, Fatemeh Yazdian, Masoud Alebouyeh, and Lobat Tayebi 
Marquette University

\section{e-Publications@Marquette}

\section{Dentistry Faculty Research and Publications/School of Dentistry}

This paper is NOT THE PUBLISHED VERSION; but the author's final, peer-reviewed manuscript. The published version may be accessed by following the link in th citation below.

Microchimica Acta, Vol. 186, No. 12 (December 2019). DOl. This article is C Springer-Verlag GmbH Austria, part of Springer Nature and permission has been granted for this version to appear in $\underline{\mathrm{e}-}$ Publications@Marquette. Springer-Verlag does not grant permission for this article to be further copied/distributed or hosted elsewhere without the express permission from Springer-Verlag.

\section{A Glassy Carbon Electrode Modified with Reduced Graphene Oxide and Gold Nanoparticles for Electrochemical Aptasensing Of Lipopolysaccharides from Escherichia Coli Bacteria}

Mehrab Pourmadadi

Protein Research Center, Shahid Beheshti University, Tehran, GC 1983963113, Iran Javad Shabani Shayeh

Protein Research Center, Shahid Beheshti University, Tehran, GC 1983963113, Iran

Meisam Omidi

Protein Research Center, Shahid Beheshti University, Tehran, GC 1983963113, Iran

Fatemeh Yazdian

Department of Life Science Engineering, Faculty of New Science and Technologies, University of Tehran, Tehran 1417466191, Iran

Masoud Alebouyeh 
Pediatric Infections Research Center, Research Institute for Children's Health, Shahid Beheshti University of Medical Sciences, Tehran 19839-63113, Iran

\section{Lobat Tayebi}

Marquette University School of Dentistry, Milwaukee, WI

\section{Abstract}

An electrochemical aptasensor is described for the voltammetric determination of lipopolysaccharide (LPS) from Escherichia coli 055:B5. Aptamer chains were immobilized on the surface of a glassy carbon electrode (GCE) via reduced graphene oxide and gold nanoparticles (RGO/AuNPs). Fast Fourier transform infrared, $\mathrm{X}$-ray diffraction and transmission electron microscopy were used to characterize the nanomaterials. Cyclic voltammetry, square wave voltammetry and electrochemical impedance spectroscopy were used to characterize the modified GCE. The results show that the modified electrode has a good selectivity for LPS over other biomolecules. The hexacyanoferrate redox system, typically operated at around $0.3 \mathrm{~V}(\mathrm{vs} . \mathrm{Ag} / \mathrm{AgCl})$ is used as an electrochemical probe. The detection limit is $30 \mathrm{fg} \cdot \mathrm{mL}^{-1}$. To decrease the electrochemical potential for detection of LPS, $\mathrm{Mg} / \mathrm{carbon}$ quantum dots were used as redox active media. They decrease the detection potentialto $0 \mathrm{~V}$ and the detection of limit (LOD) to $1 \mathrm{fg} \cdot \mathrm{mL}^{-1}$. The electrode was successfully used to analyze serum of patients and healthy persons.

\section{Keywords}

Magnesium carbon quantum dot, Hexacyanoferrate, Voltammetric determination, Real sample

\section{Introduction}

Lipopolysaccharide (LPS) is one of the main components at the outer wall membrane of all gramnegative bacteria. LPS molecules also known as endotoxin in some sources that can be found in complex environments such as the human body, food, and water. LPS has high chemical stability that after the death of gram-negative bacterial cells, as well as some live bacteria are secreted and released into the environment [[ 1]-[3]]. Due to high thermal stability of LPS is not removed when the organism is killed by the sterilization process and release of LPS occurs through the proliferation of cells [[ 4]]. The LPS molecule reaches the human immune system through the lung and caused to septic shock that is the first cause of death in intensive care unit (ICU). Therefore, high mortality rates due to shock induced by endotoxin is still a major clinical problem, the [[ 6]]. The USA Food and Drug Administration (FDA) recommends that the limit of endotoxin concentration for medical devices must be 0.5 EU. $\mathrm{mL}^{-1}$ (endotoxin unit, $1 \mathrm{EU}=0.1 \mathrm{ng}$ ) and this concentration for devices in contact with the cerebrospinal fluid and interactive medications must be $0.06 \mathrm{EU} . \mathrm{mL}^{-1}$ and $0.2 \mathrm{EU} .(\mathrm{kg} . \mathrm{hr})^{-1}$, respectively [[ 8]]. A common method for LPS detection is Limulus Amebocyte Lysate (LAL) that is based on the clotting of the crayfish cells by the endotoxin in the sample, which has been used for many years, however, this is a long time and complex process that requires an enzyme reaction to clot and this procedure is sensitive to proteases and impurities during the enzymatic reaction [[ 9]]. Therefore, preparing a fast and cheap method for detection of LPS in necessary. Nowadays, electrochemical biosensors due to their applications, ease of synthesis and usability attract more attentions [[10]]. One of the main part of electrochemical biosensors is the bioelement that be used to specification of 
targets. Anti-bodies, enzyme, cell, aptamer and etc. are some kind of bioelements that among of them, aptamer due to stability, selectivity and their cost have been selected recently [[12]-[14]]. Aptamers are synthetic single-stranded oligonucleotides that synthesized by Systematic evolution of ligands by exponential enrichment (SELEX) process, used for fabrication a wide range of biosensors. [[15]-[17]]. There are many reports that used aptamer as bioelement to detect various materials such as biomacromolecules such as LPS [[18]-[20]] and heavy metals [[21]]. One of the most important parameters in aptasensor preparing is the electrode modification procedure in that aptamer chains decorated on the surface of electrode [[14]]. This step is very critical because can be deactivate the performance of aptasensor [[22]]. Use of nanomaterials can help to simple and fast modification of aptasensor electrodes that among of all nanomaterials, graphene has many advantages against the others due to high surface area, chemical stability, electrical conductivity and other benefits [[23]]. There are some methods for synthesis of graphene that hummer method due to simple procedure have been chosen for researchers. In this procedure, graphite convert to GO and after that transform to reduced graphene oxide that called RGO. Although the electrical conductivity of RGO is lower than graphene but using some metal nanoparticles can improve the RGO properties [[24]-[26]]. RGO sheets that modified by AuNPs have suitable electrical conductivity and can used for decoration of thiolated biomarkers such as aptamer targets [[27]]. In this work, we present an aptasensor for LPS detection using RGO/AuNPs for fast immobilization of aptamer chains on the surface of electrode. For this purpose, RGO/AuNPs was synthesized chemically and after that aptamer chains connected to graphene sheets through EDC/NHS - AuNPs bond. At the next step, determination of LPS macromolecules were studied in hexacyanoferrate and $\mathrm{Mg} / \mathrm{CQD}$ media.

\section{Materials and apparatus}

Analytical grade graphite powder, Sodium borohydride $\left(\mathrm{NaBH}_{4}\right), \mathrm{HAuCl}_{4} .4 \mathrm{H}_{2} \mathrm{O}$, potassium hexacyanoferrate (II) ( $\left.\mathrm{K}_{4}\left[\mathrm{Fe}(\mathrm{CN})_{6}\right]\right)$, Diammonium Hydrogen Citrate $\left(\mathrm{C}_{6} \mathrm{H}_{14} \mathrm{~N}_{2} \mathrm{O}_{7}\right), \mathrm{MgCl} .{ }_{2} \mathrm{H}_{2} \mathrm{O}$, 1-Ethyl-3(3-dimethylaminopropyl) carbodiimide (EDC), N-Hydroxysuccinimide (NHS), fetal bovine serum (FBS), glucose and bovine serum albumin (BSA) were all purchased from Sigma-Aldrich (Germany http://www.sigmaaldrich.com), and were used as received. All solutions were prepared by double distilled water. Extracted LPS from Escherichia coli 055:B5 (L4524) with the molecular weight of 390 kDa, was purchased from sigma aldrich (USA, http://www.sigmaaldrich.com).

The LPS aptamer $(\mathrm{Kd}=12 \mathrm{nM})$ with $6{ }^{\prime} \mathrm{NH}_{2}$ modification and HPLC purification was synthesized by bio basic inc. (Canada, https://www.biobasic.com) with the following sequence [[ 8], [28]]:

(CTTCTGCCCGCCTCCTTCCTAGCCGGATCGCGCTGGCCAGATGATATAAAGGGTCAGCCCCCCAGGAGACGAGA TAGGCGGACACTOD5 HPLC 5' Mod Amino Modifier - $\mathrm{NH}_{2} \mathrm{C}_{6}$ ). The lyophilized aptamer powder was diluted by phosphate buffer saline (PBS), $(\mathrm{NaCl} 0.138 \mathrm{M} ; \mathrm{KCl}-0.0027 \mathrm{M}) ; \mathrm{pH}=7.4$ and restored at -20 ${ }^{\circ} \mathrm{C}$. The electrochemical experiments were done using a potentiostat/galvanostat device designed by Ivium technologies, (Netherlands https://www.ivium.com) in ferricyanide redox probe $0.2 \mathrm{mM}$ and PBS $0.1 \mathrm{M}$. A three electrode system consisted of a glassy carbon electrode (diameter $=2 \mathrm{~mm}$ ) as working electrode, a Platinum electrode and an $\mathrm{Ag} / \mathrm{AgCl}$ electrode as counter and reference, respectively (Detectco https://www.detectco.com). The EIS experiments were conducted in the frequency range between $100 \mathrm{kHz}$ and $15 \mathrm{mHz}$ with perturbation amplitude of $5 \mathrm{mV}$ at the potential of oxidation peak current of CV curves. 
Morphological investigations of the polymeric films were carried out by using SEM (Philips XL 30 and KYKY-EM3200, https://www.semtechsolutions.com) and transmission electron microscopy (TEM, Philips Tecnai G220, operated at 120 kV, https://www.jeolusa.com/). XRD measurements were performed using an X-ray diffractometer (STOE, Germany https://www.stoe.com) with a Cu-Ka monochromatized radiation source and a Ni filter. FT-IR spectra were obtained using a spectrometer (Thermo Incol, USA; https://www.thermofisher.com). Dynamic light scattering (DLS) was done on a (Nano Phox, Germany https://www.sympatec.com) instrument. All measurements were performed at room temperature.

\section{Synthesis of RGO/au nanocomposite}

Graphene oxide was synthesized chemically by Hummer method [[29]]. For synthesis of RGO/AuNPs, $0.6 \mathrm{~g} \mathrm{GO}$ was added to $100 \mathrm{~mL} \mathrm{H} \mathrm{H}_{2} \mathrm{O}$ in sonication bath for $10 \mathrm{~min}$ and after that $1 \mathrm{~g} \mathrm{NaBH}_{4}$ was added to suspension slowly and the mixture stirred for $3 \mathrm{~h}$. The RGO that produced in this reaction must be dried at $80^{\circ} \mathrm{C}$ for $24 \mathrm{~h}$. Then, $0.35 \mathrm{~g}$ of RGO was added to $80 \mathrm{~mL} \mathrm{H}_{2} \mathrm{O}$ in sonication bath for $10 \mathrm{~min}$ and after that $2 \mathrm{~mL} \mathrm{HAuCl} 4.6 \mathrm{H}_{2} \mathrm{O} 0.25 \mathrm{M}$ dropped to mixture and stirred for $3 \mathrm{~h}$. After that the product washed by $\mathrm{H}_{2} \mathrm{O}$ and $\mathrm{C}_{2} \mathrm{H}_{5} \mathrm{OH}$ and dried at $80{ }^{\circ} \mathrm{C}$ for $24 \mathrm{~h}$.

\section{Synthesis of mg/CQDs}

Di-Ammonium hydrogen citrate $\left(2 \mathrm{~g}\right.$ ) as carbon source solved in $75 \mathrm{~mL} \mathrm{H}_{2} \mathrm{O}$ at room temperature and then $1 \mathrm{~g} \mathrm{MgCl}_{2} .6 \mathrm{H}_{2} \mathrm{O}$ was added and stirred until solved. After that $0.4 \mathrm{~g} \mathrm{NaOH}$ as stabilizer was added to solution, then the solution inserted in a hydrothermal bomb and kept at $150{ }^{\circ} \mathrm{C}$ for $24 \mathrm{~h}$.

\section{Incubation and preparing the RGO/AuNPs-apt}

$100 \mu \mathrm{L}$ EDC $0.5 \mathrm{mg} \cdot \mathrm{mL}^{-1}$ mixed with $100 \mu \mathrm{L} \mathrm{NHS} 0.5 \mathrm{mg} \cdot \mathrm{mL}^{-1}$ and shake for $15 \mathrm{~min}$, then $200 \mu \mathrm{L}$ RGO/AuNPs ( $\left.0.25 \mathrm{mg} \cdot \mathrm{mL}^{-1}\right)$ was added to solution and shake for $15 \mathrm{~min}$. After that $2 \mu \mathrm{L}$ aptamer (100 $\mu \mathrm{M}$ ) was added and solution shake for $3 \mathrm{~h}$. Finally, the mixture centrifuged at $6000 \mathrm{RPM}$ and the upper solution was wasted till RGO/AuNPs-apt resulted.

\section{Electrode modification procedure}

$4 \mu \mathrm{L}$ RGO/AuNPs-apt $\left(0.25 \mathrm{mg} \cdot \mathrm{mL}^{-1}\right)$ was mixed by $0.1 \%$ wt. nafion (1:10) for $15 \mathrm{~min}$ and after that this volume dropped on the surface of GC electrode until dried at room temperature. For all electrochemical tests $10 \mu \mathrm{L}$ from the samples dropped on the surface of modified electrode for selected time and after that the electrode rinsed in PBS $(\mathrm{pH}=7.4)$ to release unbonded materials at the surface of electrode. Finally the modified electrode was ready to electrochemical analysis.

\section{Real sample analysis}

5 serum samples including three positive control (patient sample) and two negative control (normal sample) samples that determined by E-TOXATE (The E-TOXATE (Limulus Amebocyte Lysate) test kits purchased from Sigma-Aldrich that is intended for semiquantitative detection of endotoxins for research purposes were used. Both real patient and normal samples were diluted 100, 300, 500, 700 and 900 times to prevent the effect of confounding factors and placed $10 \mu \mathrm{L}$ of the lowest concentration of real samples as analyte on the electrode surface, followed by incubation with specific 
aptamer for 35 min as same as synthetic samples protocol. Then washed by addition PBS with $\mathrm{pH}=7.4$ to remove unbound lipopolysaccharide similarly for subsequent concentrations of low to high concentrations for all 5 real negative control and positive control samples.

\section{Results and discussion}

Figure 1 presents the structural analysis of synthesized materials. X-Ray pattern of GO shows a sharp peak at $10^{\circ}$ reflect to (111) GO sheets and the broad peak at about $20^{\circ}$ is related to un oxidized graphite sheets. Reduction of GO and decoration of sheets with Au nano particles caused to appearance of new reflection analysis in RGO/Au NPs XRD pattern(Fig. 1.a). as can be seen, reduction of GO and presence of AuNPs caused to appearance of some sharp diffraction peaks at 38.14, 44.32, 64.66 and 77.74 that related to (200), (111), (220) and 311 for AuNPs. XRD pattern of CQD and $\mathrm{Mg} / \mathrm{CQD}$ presented in Fig. 1b. The diffraction peak displays a broad peak centered at 24 correspond to amorphous nature of the system. By incorporation of $\mathrm{Mg}$ in the structure of CQD a sharp peak at 31 and 49 were appeared matched to standard pattern JCPDs \#00-041-1445 [[30]]. FTIR analysis has many information about the structural of synthesized materials illustrated in Fig. 1c. FTIR spectrum of $\mathrm{GO}$ shows an absorption band at 3360 and $1040 \mathrm{~cm}^{-1}$ correspond to $\mathrm{OH}$ groups and $\mathrm{C}-\mathrm{O}$, respectively. Furthermore an absorption peak at $1710 \mathrm{~cm}^{-1}$ related to $\mathrm{C}=\mathrm{O}$ functional groups that can react with other functional groups biomaterials such as aptamer chains. By reduction of $\mathrm{GO}$ sheets and $\mathrm{C}=\mathrm{O}$ groups also the electronic structure of carbon atoms change from $\mathrm{SP}^{3}$ to $\mathrm{SP}^{2}$ and therefore magnitude of $\mathrm{C}=\mathrm{C}$ bonds increased that appeared at RGO/AuNPs spectrum. After aptamer bonding on the surface of RGO/AuNPs, absorption peaks related to O-H and C-O groups decreased, presented in RGO/AuNPsaptamer spectrum. Figure $1 d$, shows the FT-IR pattern of CQD and Mg/CQD. The peaks about 3100 $\mathrm{cm}^{-1}$ and $1640 \mathrm{~cm}^{-1}$ in both graphs related to the stretching and deformation mode of $-\mathrm{O}-\mathrm{H}$ functional group, respectively. The peaks at 3190,1570, 1410 and $1250 \mathrm{~cm}^{-1}$ are attributed to the $-\mathrm{N}-$ $\mathrm{H},-\mathrm{C}=\mathrm{O},-\mathrm{C}=\mathrm{C}$ and $-\mathrm{C}-\mathrm{N}$ stretching vibration, respectively. Presence of oxygen atoms on surface and interaction with $\mathrm{Mg}$ atoms ( $\mathrm{Mg}-\mathrm{O}$ surf) create a small peak at $1100 \mathrm{~cm}^{-1}$ whereas the metal oxide peaks for $\mathrm{MgO}$ resulted about $500-600 \mathrm{~cm}^{-1}$ correspond to asymmetric stretching of $\mathrm{Mg}-\mathrm{O}-\mathrm{Mg}$. The TEM image of RGO/Au NPs presents at Fig. 1c. As can be seen, Au NPs distributed on the surface of RGO Sheets uniformly. The average size of AuNPs was estimated $50 \mathrm{~nm}$ approximately. Modification of RGO sheets by Au nano particles caused to increase the conductivity of RGO sheets and furthermore Au NPs provide a suitable structure for aptamer decoration.

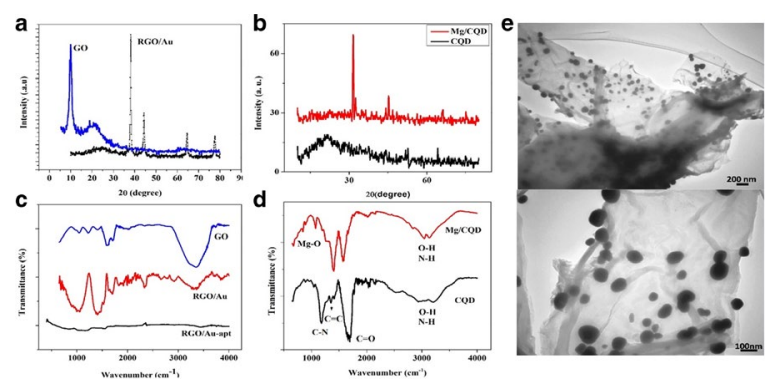

Fig. 1 XRD patterns of GO and RGO/Au NP (a) CQD and Mg/CQD (b) FTIR spectra of GO, RGO/Au NPs and RGO/Au NPs-aptamer (c) CQD and Mg/CQD (d) and TEM graphs of RGO/Au NPs (e)

The modification process of electrode was studied by electrochemical techniques. Figure 2a shows the CV curves of GCE after each modification step. Modification of GCE surface with RGO/Au NPs caused to 
increase the active surface area of electrode and therefore the current peaks of $\left[\mathrm{Fe}(\mathrm{CN})_{6}\right]^{-3 /-4}$ reaction enhanced. By immobilization of aptamer chains and addition of nafion as a blocker on the surface of GCE/ RGO/Au NPs some of active sites blocked that resulted to decrease the peak current of CV curves, respectively. Furthermore, after folding procedure between LPS and aptamer chains due to large size of LPS macromolecules, the peak current of $\left[\mathrm{Fe}(\mathrm{CN})_{6}\right]^{-3 /-4}$ reaction decreased suddenly. EIS is one of the electrochemical techniques that provide useful information about charge transfer on the electrode interface [[31]-[33]]. Figure $2 \mathrm{~b}$ presents the Nyquist graphs of electrode modification process. As illustrated, each graph has a semi-circle that related to the charge transfer resistance $\left(R_{c t}\right)$ of [Fe $\left.(\mathrm{CN})_{6}\right]^{-3 /-4}$ reaction that appeared to diameter of semicircles. By modification of GCE surface with various materials, the surface resistance of electrode varied and therefore the diameter increased except the RGO/AuNPs modification step that due to increase the conductivity and surface area of electrode the diameter of Nyquist diagrams decreased. After modification process, the main concept of LPS determination is the optimum time. The time profile of nanoprobe electrode in $\left[\mathrm{Fe}(\mathrm{CN})_{6}\right]^{-}$ ${ }^{3 /-4}$ media at the concentration of $0.5 \mathrm{pg} \cdot \mathrm{mL}^{-1}$ presented in Fig. $2 \mathrm{c}$. As shows, the response of electrode after 35 min get stable that shows the optimum time for determination of LPS is $35 \mathrm{~min}$.
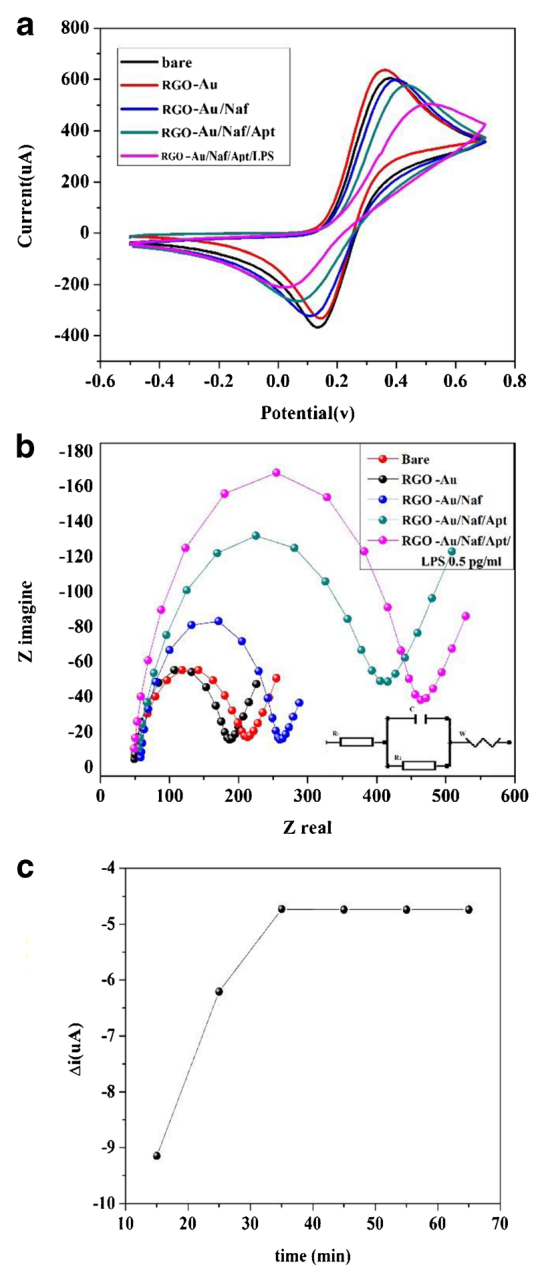

Fig. 2 The electrochemical characterization of the nanocomposite a The CV analysis at the scan rate of $50 \mathrm{mV} \mathrm{s}-1$ and $b$ The EIS analysis of the bare and each step of modified electrodes in [Fe(CN)6] $-3 /-4$ $(0.2 \mathrm{mM})$, c time profile of aptamer/LPS interaction based on SWV techniques at different incubation time 
Figure 3a Presents the SW voltammograms of nanoprobe electrode in $\left[\mathrm{Fe}(\mathrm{CN})_{6}\right]^{-3 /-4}$ media at various concentration of LPS. As can be seen, after each folding process, due to surface occupation of LPS molecules, the peak current decreased that correspond from a linear behavior, presented in Fig. S2a. Nyquist diagrams of nanoprobe electrode in $\left[\mathrm{Fe}(\mathrm{CN})_{6}\right]^{-3 /-4}$ media has a semi-circle with a diameter related to charge transfer resistance of $\left[\mathrm{Fe}(\mathrm{CN})_{6}\right]^{-3 /-4}$ reaction. By increasing the concentration of LPS at the surface of electrode and folding process, the surface resistance of electrode increase and therefore, the $\mathrm{R}_{\mathrm{ct}}$ increased (Fig. 3c). Linear behavior of nanoprobe electrode at various concentration of LPS described the suitable capability of LPS detection (Fig. S2b). LOD describe the performance of a nanoprobe electrode that calculated following

(1) LOD $=\frac{3 s b}{m}$
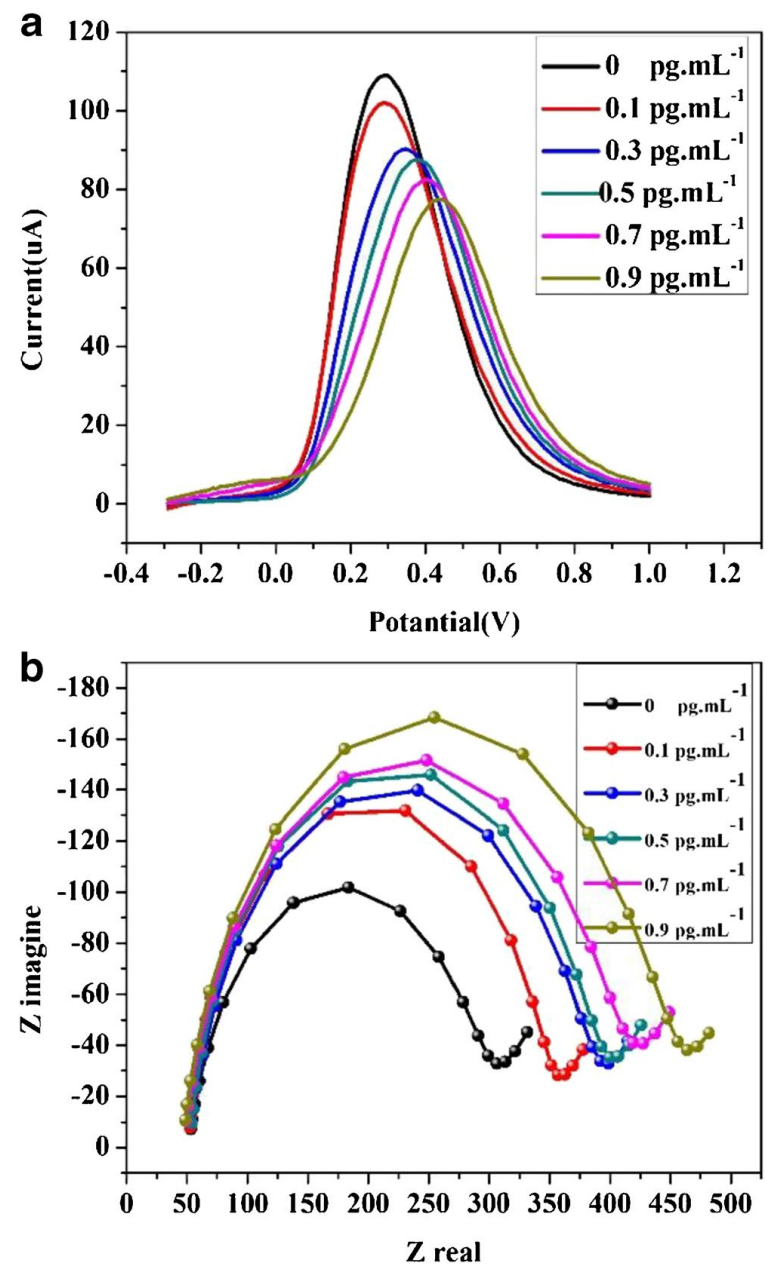

Fig. 3 a SW voltammograms of GCE/ RGO/Au NPs-aptamer electrode, b Nyquist diagrams of electrode in [Fe (CN)6]3-/4- media at various concentration of LPS

Where $S_{b}$ is the standard deviation for blank and $m$ is the slope of calibration plot. The magnitude of LOD using Eq. 1 was obtained about $0.03 \mathrm{pg} \cdot \mathrm{mL}^{-1}$. Due to same order of calibration plots of SWV and EIS techniques the magnitude of calculated LOD for both are similar together, approximately.

One of the most important parameters about the biosensors is to be user-friendly. Although Potassium ferrocyanide has a good electrochemical signal but is hazardous. For this reason and study the application of nanoprobe electrode in PBS media we used Mg/CQD as an electrochemical mediator. 
Fig. S3a presents the 50 continues CV curves of nanoprobe electrode in $10 \mathrm{mM}$ PBS media contained $\mathrm{Mg} / \mathrm{CQD}\left(40 \mu \mathrm{g} \cdot \mathrm{mL}^{-1}\right)$ at the scan rate of $50 \mathrm{mV} / \mathrm{s}$. As can be seen, Each CV curves has an oxidation peak at $0 \mathrm{~V}$ and a broad reduction peak around -0.1 to $-0.4 \mathrm{~V}$. Continues cycles illustrated that $\mathrm{Mg} / \mathrm{CQDs}$ cannot be accumulated on the electrode. CV curves of RGO/AuNPs-aptamer in Mg/CQD media at various concentrations of LPS shows that by increasing the concentration of macromolecules at the electrode surface due to blocking the active sites, the redox peak current decreased (Fig. S3b). The second oxidation peak appears in CV curves at negative potentials about $-0.3 \mathrm{~V}$ can be related to interaction of LPS macromolecules and Mg/CQDs. Size of Mg/CQDs is another feature of this nanomaterials that by DLS analysis the size of this CQDs was calculated about $75 \mathrm{~nm}$ (Fig. 4a). To study the interaction between PBS, PBS/apt, Mg.CQDs, Ultraviolet-visible with 250 to $950 \mathrm{~nm}$ wavelength was performed (Fig. 4b). As can be seen in Fig. 4b their absorption between 500 to $950 \mathrm{~nm}$ (zoom out area) were completely different, so that, PBS and PBS/ Apt as blank, have not obvious absorption but Mg.CQDs has a sharp absorption in this area, on the other hand, by addition of LPS to Mg.CQDs, absorption was increased by concentration against Mg.CQDs that means LPS has interaction with Mg.CQDs and also, when was tested Mg.CQDs/apt with the same various concentration of LPS, absorptions were increased again that shows the interaction between LPS with aptamer in the presence of Mg.CQDs. All of these results approved the appearance of the second peak in electrochemical tests. Figure 4c, presents the SW voltammograms of RGO/AuNPs-apt electrode in Mg.CQDs media at various concentration of LPS. According to CV reults, each curve has two oxidative peak that by increasing the LPS concentration and interaction with aptamer chains, due to blocking the active sites, the magnitude of peak current decreased. The LOD of the biosensor electrode was calculated at about $1 \mathrm{fg} \cdot \mathrm{mL}^{-1}$. The results show that $\mathrm{Mg} / \mathrm{CQDs}$ provides a suitable media for determination of LPS with some benefits (Fig. 4d). Using Mg/CQDs has two benefits, first is a determination of LPS in PBS media that is very close to human blood and latter is the potential for electrochemical detection that is about $0 \mathrm{~V}$.
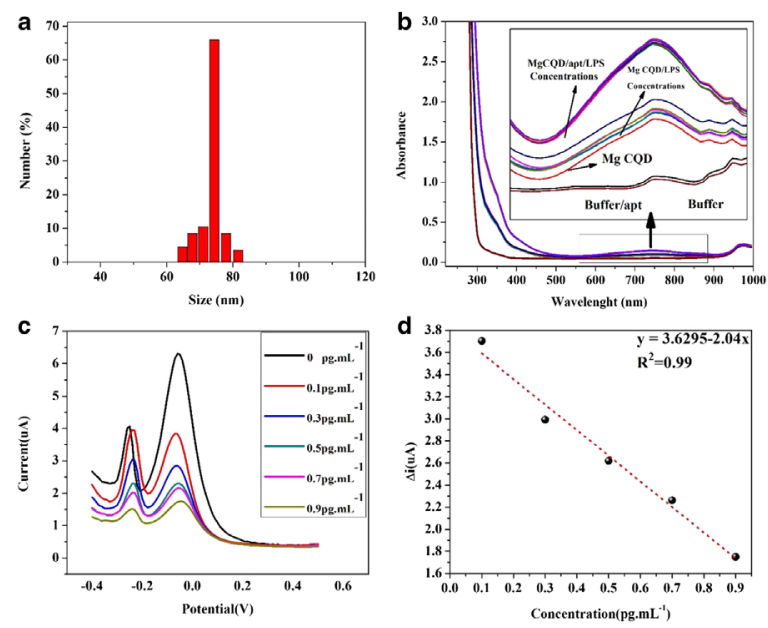

Fig. 4 a DLS analysis of $\mathrm{Mg} / \mathrm{CQDs}(40 \mu \mathrm{g} / \mathrm{ml}) \mathrm{b}$ Ultraviolet-visible absorption of the $\mathrm{Mg}$.CQDs at various concentration of LPS[0.1, 0.3, 0.5, $0.7,0.9 \mathrm{pg} . \mathrm{mL}]$ and Mg.CQDs/apt/ various concentration of LPS[0.1, $0.3,0.5,0.7,0.9 \mathrm{pg} . \mathrm{mL}]$, c SW voltammograms of nano probe electrode in $\mathrm{Mg} / \mathrm{CQD}(40 \mu \mathrm{g} / \mathrm{ml})$ media at various concentrations of LPS, d calibration plot derived from SWV

Usability of a nanoprobe at medical aims is the next step in nanobiotechnology studies. Fig. S4, presents the SW voltammograms of a patient and a healthy person in PBS/Mg CQD media. As can be 
seen, each voltammogram has two oxidation peak as same as synthetic samples that by increasing the sample dilution, the second peak of SW voltammogram of positive sample decreased and show a logic pattern, whereas this behavior wasn't seen in normal person serums. Figure 5(a-e) presents the electrochemical signal of nanoprobe electrode for 3 patient person and two healthy one. As can be seen, the signal pattern of patients has a logic form that the signal of electrode decreased when the dilution of people serum increased. The behavior of electrode for three patients is same as together whereas for healthy persons the pattern of signal - concentration profile is not logic and same together. The results show the electrochemical nanoprobe can be used at medical purpose by this form. Although LAL is a trustable method to LPS detection, but the quantity measurement of LPS by LAL method is not reported. RGO/AuNPs-aptamer electrode has the capability of LAL detection with low LOD order in that determination in Mg/CQDs - PBS media has more benefits. Figure $5 f$. Presents the selectivity of nanoprobe electrode. The modified electrode has an impressive response against other biomaterials that presence in human blood and emphasize that RGO/AuNPs-aptamer electrode is a suitable nanoprobe for LPS detection. Comparison of prepared nanoprobe electrode with other reported electrodes presented in Table 1. The results show the suitable performance of prepared nanoprobe against other reports.

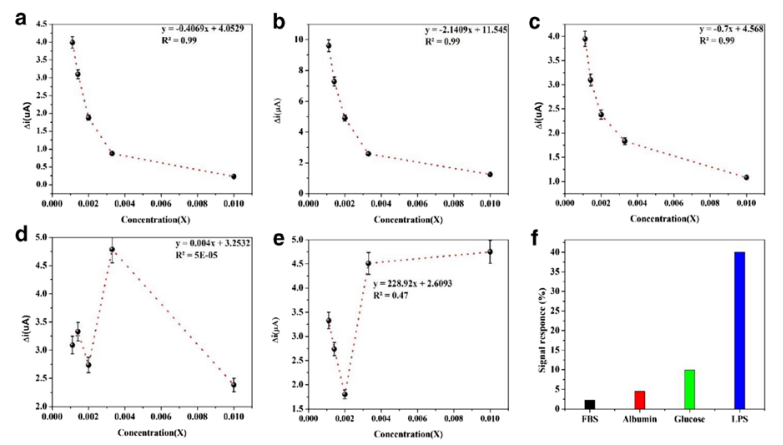

Fig. 5 a Current - serum dilution profile for patient No.1, b patient No.2, c patient No.3, d healthy No.1, e healthy No.2, $\mathrm{f}$ comparsion of nano probe electrode signal response for LPS (0.5 pg.mL-1), glucose (90 mg.dL-1), Albumin (7 $\mu \mathrm{g} . \mathrm{mL}-1)$ and FBS (5 ng.mL-1) 
Table 1. Comparison of the analytical performance of the new nano probe with other electrochemical lipopolysaccharide nano probes

\begin{tabular}{|c|c|c|c|}
\hline Electrode material & $\begin{array}{l}\text { Linear range (as } \\
\text { reported) }\end{array}$ & Detection limit (as reported) & References \\
\hline $\begin{array}{l}\text { Reduced graphene oxide/ Gold Nanoparticles/GC } \\
\text { electrode }\end{array}$ & $\left(0.1-0.9\right.$ pg. $\left.\mathrm{mL}^{-1}\right)$ & $1 \mathrm{fg} \cdot \mathrm{mL}^{-1}$ & The present Work \\
\hline Gold Nanoparticles/PEDOT/ Gold electrode & $0.1-1000$ pg.mL $\mathrm{mL}^{-1}$ & 0.1 pg. $\mathrm{mL}^{-1}$ & {$[8]$} \\
\hline Apt/ MPA / Gold electrode & $1-1000 \mathrm{pg} \cdot \mathrm{mL}^{-1}$ & $1 \mathrm{pg} \cdot \mathrm{mL}^{-1}$ & {$[13]$} \\
\hline $\begin{array}{l}\text { 6-mercapto-1-hexanol (MCH)-blocked aptamer/AuNP- } \\
\text { coated }\end{array}$ & 0.01 to10.24 ng.mL $\mathrm{mL}^{-1}$ & $0.005 \mathrm{ng} \cdot \mathrm{mL}^{-1}$ & {$[28]$} \\
\hline Apt/gold atomic clusters/ Gold electrode & $0.01 \mathrm{aM}$ to $1 \mathrm{pM}$ & $7.94 \times 10^{-21} \mathrm{M}$ & {$[34]$} \\
\hline Apt/ MCH / Gold electrode & 0.01 to $1 \mathrm{ng} \cdot \mathrm{mL}^{-1}$ & $0.001 \mathrm{ng} \cdot \mathrm{mL}^{-1}$ & {$[35]$} \\
\hline
\end{tabular}




\section{Conclusion}

In this work we presents an electrochemical nanoprobe electrode for detection of LPS. The designed electrode shows a suitable selectivity to LPS among other biomaterials and lower LOD against previous reports. Using Mg/CQDs as a new media caused to detect LPS in PBS at very low potentials that clear the benefit of using quantum dots. Decoration of reduced graphene oxide with Au/NPs caused to fast aptamer immobilization on the surface of electrode and increased the electrochemical conductivity of reduced graphene oxide sheets. Comparison of designed nanoprobe with LAL method for patient and healthy peoples shows that this electrode is a good candidate for LPS detection in medical purpose.

\section{Electronic supplementary material}

Graph: (DOCX $936 \mathrm{~kb}$ )

\section{Publisher's note}

Springer Nature remains neutral with regard to jurisdictional claims in published maps and institutional affiliations.

\section{References}

1. Miao P. Electrochemical sensing strategies for the detection of endotoxin: a review. RSC Adv. 2013; 3: 9606-9617. 10.1039/c3ra00047h

2. Wright SD, Ramos RA, Tobias PS, Ulevitch RJ, Mathison JC. CD14, a receptor for complexes of lipopolysaccharide (LPS) and LPS binding protein. Science. 1990; 249; 4975: 1431-1433. 10.1126/science.1698311

3. Opal SM, Scannon PJ, Vincent J-L, White M, Carroll SF, Palardy JE, Parejo NA, Pribble JP, Lemke $\mathrm{JH}$. Relationship between plasma levels of lipopolysaccharide (LPS) and LPS-binding protein in patients with severe sepsis and septic shock. J Infect Dis. 1999; 180: 1584-1589. $10.1086 / 315093$

4. Lan M, Wu J, Liu W, Zhang W, Ge J, Zhang H, Sun J, Zhao W, Wang P. Copolythiophene-derived colorimetric and fluorometric sensor for visually supersensitive determination of lipopolysaccharide. JACS. 2012; 134: 6685-6694. 10.1021/ja211570a

5. de La Lastra CA, Villegas I, Sanchez-Fidalgo S. Poly (ADP-ribose) polymerase inhibitors: new pharmacological functions and potential clinical implications. Curr Pharm Des. 2007; 13: 933962. 10.2174/138161207780414241

6. A-q W, Yang Q-w, J-c L, F-I L, Zhong Q, C-y C. A novel lipopolysaccharide-antagonizing aptamer protects mice against endotoxemia. Biochem Biophys Res Commun. 2009; 382: 140-144. 10.1016/j.bbrc.2009.02.152

7. Zhao Y, Cong L, Jaber V, Lukiw WJ. Microbiome-derived lipopolysaccharide enriched in the perinuclear region of Alzheimer's disease brain. Front Immunol. 2017; 8: 1064. 10.3389/fimmu.2017.01064

8. Su W, Cho M, Nam JD, Choe WS, Lee Y. Aptamer-assisted gold nanoparticles/PEDOT platform for ultrasensitive detection of LPS. Electroanalysis. 2013; 25: 380-386. 10.1002/elan.201200453 
9. Sakti SP, Lucklum R, Hauptmann P, Bühling F, Ansorge S. Disposable TSM-biosensor based on viscosity changes of the contacting medium. Biosens Bioelectron. 2001; 16: 1101-1108. 10.1016/S0956-5663(01)00190-7

10. Wang J. Carbon-nanotube based electrochemical biosensors: A review. Electroanalysis. 2005; 17: 7-14. 10.1002/elan.200403113

11. Shao Y, Wang J, Wu H, Liu J, Aksay IA, Lin Y. Graphene based electrochemical sensors and biosensors: a review. Electroanalysis. 2010; 22: 1027-1036. 10.1002/elan.200900571

12. Calderón K, Martín-Pascual J, Poyatos JM, Rodelas B, González-Martínez A, González-López J. Comparative analysis of the bacterial diversity in a lab-scale moving bed biofilm reactor (MBBR) applied to treat urban wastewater under different operational conditions. Bioresour Technol. 2012; 121: 119-126. 10.1016/j.biortech.2012.06.078

13. Su W, Lin M, Lee $H$, Cho M, Choe W-S, Lee Y. Determination of endotoxin through an aptamerbased impedance biosensor. Biosens Bioelectron. 2012; 32: 32-36. 10.1016/j.bios.2011.11.009

14. Zuo M-Y, Chen L-J, Jiang H, Tan L, Luo Z-F, Wang Y-M. Detecting endotoxin with a flow cytometry-based magnetic aptasensor. Anal Biochem. 2014; 466: 38-43. 10.1016/j.ab.2014.08.018

15. Zhao W, Chiuman W, Lam JC, McManus SA, Chen W, Cui Y, Pelton R, Brook MA, Li Y. DNA aptamer folding on gold nanoparticles: from colloid chemistry to biosensors. JACS. 2008; 130: 3610-3618. 10.1021/ja710241b

16. Anthony PC, Perez CF, García-García C, Block SM. Folding energy landscape of the thiamine pyrophosphate riboswitch aptamer. PNAS USA. 2012; 109: 1485-1489. 10.1073/pnas.1115045109

17. Frieda KL, Block SM. Direct observation of cotranscriptional folding in an adenine riboswitch. Science. 2012; 338: 397-400. 10.1126/science.1225722

18. Xu W, Tian J, Shao X, Zhu L, Huang K, Luo Y. A rapid and visual aptasensor for lipopolysaccharides detection based on the bulb-like triplex turn-on switch coupled with HCRHRP nanostructures. Biosens Bioelectron. 2017; 89: 795-801. 10.1016/j.bios.2016.10.012

19. Bai L, Chai Y, Pu X, Yuan R. A signal-on electrochemical aptasensor for ultrasensitive detection of endotoxin using three-way DNA junction-aided enzymatic recycling and graphene nanohybrid for amplification. Nanoscale. 2014; 6: 2902-2908. 10.1039/c3nr05930h

20. Chen C, Xie Q, Yang D, Xiao H, Fu Y, Tan Y, Yao S. Recent advances in electrochemical glucose biosensors: a review. RSC Adv. 2013; 3: 4473-4491. 10.1039/c2ra22351a

21. An JH, Park SJ, Kwon OS, Bae J, Jang J. High-performance flexible Graphene Aptasensor for mercury detection in mussels. ACS Nano. 2013; 7: 10563-10571. 10.1021/nn402702w

22. Shen W-J, Zhuo Y, Chai Y-Q, Yuan R. Cu-based metal-organic frameworks as a catalyst to construct a Ratiometric electrochemical Aptasensor for sensitive lipopolysaccharide detection. Anal Chem. 2015; 87; 22: 11345-11352. 10.1021/acs.analchem.5b02694

23. Feng L, Chen Y, Ren J, Qu X. A graphene functionalized electrochemical aptasensor for selective label-free detection of cancer cells. Biomaterials. 2011; 32: 2930-2937. 10.1016/j.biomaterials.2011.01.002

24. Shabani Shayeh J, Nikkar A, Norouzi P, Ganjali MR, Wojdyla M. Physioelectrochemical investigation of supercapacitive performance of ternary nanocomposite by common 
electrochemical methods and fast fourier transform voltammetry. New J Chem. 2015; 39: 94549460. 10.1039/C5NJ01954K

25. Shayeh JS, Ehsani A, Ganjali M, Norouzi P, Jaleh B. Conductive polymer/reduced graphene oxide/au nano particles as efficient composite materials in electrochemical supercapacitors. Appl Surf Sci. 2015; 353: 594-599. 10.1016/j.apsusc.2015.06.066

26. Vahidzadeh E, Fatemi S, Nouralishahi A. Synthesis of a nitrogen-doped titanium dioxidereduced graphene oxide nanocomposite for photocatalysis under visible light irradiation. Particuology. 2018; 41: 48-57. 10.1016/j.partic.2017.12.013

27. Ferapontova EE, Olsen EM, Gothelf KV. An RNA aptamer-based electrochemical biosensor for detection of theophylline in serum. JACS. 2008; 130: 4256-4258. 10.1021/ja711326b

28. Su W, Kim S-E, Cho M, Nam J-D, Choe W-S, Lee Y. Selective detection of endotoxin using an impedance aptasensor with electrochemically deposited gold nanoparticles. J Innate Immun. 2013; 19: 388-397. 10.1177/1753425912465099

29. Chen J, Yao B, Li C, Shi G. An improved hummers method for eco-friendly synthesis of graphene oxide. Carbon. 2013; 64: 225-229. 10.1016/j.carbon.2013.07.055

30. Muthusankar G, Rajkumar C, Chen S-M, Karkuzhali R, Gopu G, Sangili A, Sengottuvelan N, Sankar R. Sonochemical driven simple preparation of nitrogen-doped carbon quantum dots/SnO2 nanocomposite: A novel electrocatalyst for sensitive voltammetric determination of riboflavin. Sensors Actuators B Chem. 2019; 281: 602-612. 10.1016/j.snb.2018.10.145

31. Shayeh JS, Ehsani A, Naeemy A, Shiri HM, Fatemi F, Yadegari A, Omidi M. Electrosynthesis and characterization of poly aniline/garnet nanoparticles for high-performance electrochemical capacitors. Ionics. 2018; 24: 505-511. 10.1007/s11581-017-2217-4

32. Shayeh JS, Norouzi P, Ganjali MR. Effect of thickness on the capacitive behavior and stability of ultrathin polyaniline for high speed super capacitors. Russ J Electrochem. 2016; 52: 933-937. 10.1134/S1023193516100128

33. Shayeh JS, Salari H, Daliri A, Omidi M. Decorative reduced graphene oxide/C3N4/Ag2O/conductive polymer as a high performance material for electrochemical capacitors. Appl Surf Sci. 2018; 447: 374-380. 10.1016/j.apsusc.2018.03.249

34. Posha B, Nambiar SR, Sandhyarani N. Gold atomic cluster mediated electrochemical aptasensor for the detection of lipopolysaccharide. Biosens Bioelectron. 2017; 101: 199-205. 10.1016/j.bios.2017.10.030

35. Kim SE, Su W, Cho M, Lee Y, Choe WS. Harnessing aptamers for electrochemical detection of endotoxin. Anal Biochem. 2012; 424: 12-20. 10.1016/j.ab.2012.02.016 